\title{
Electrical and Spectroscopic Studies of the CdO Substituted Lead Vanadate Glass System vs Crystalline Form
}

\author{
Ponnada Tejeswara Rao ${ }^{1}$, Kocharlakota V. Ramesh ${ }^{1 *}$, Devulapalli L. Sastry ${ }^{2}$ \\ ${ }^{1}$ Department of Physics, GITAM Institute of Technology, GITAM University, Visakhapatnam, India; ${ }^{2}$ Department of Physics, J. V. \\ D. College of Science, Andhra University, Visakhapatnam, India. \\ Email: "kvramesh11@gmail.com,kv_ramesh5@yahoo.co.in
}

Received September $26^{\text {th }}, 2011$; revised November $6^{\text {th }}, 2011$; accepted November $16^{\text {th }}, 2011$

\begin{abstract}
Results of the direct current (DC) Electrical Conductivity, thermoelectric power and Electron Spin Resonance (ESR) of $\mathrm{CdO}$ substituted $\mathrm{PbO}-\mathrm{V}_{2} \mathrm{O}_{5}$ glass system are reported. Conduction in these glasses is found to be electronic and the hoping of polaron seems to be the dominant process in the transport mechanism. There is a remarkable decrease in the activation energy for conduction in the annealed and devitrified samples when compared to their amorphous counter parts. It is observed that there is remarkable improvement in the conductivity of the crystalline samples when compared to their amorphous counter parts. The thermoelectric power measurements indicates that the amorphous samples are n-type at room temperature where as the crystalline samples are p-type at room temperature. In crystalline samples the hyperfine structure is nearly smeared out and a relatively broad line with an isotropic $g$ value characterizes the spectra.
\end{abstract}

Keywords: Transport Properties-ESR; Lead Vanadate; Semi Conducting Glasses

\section{Introduction}

The $\mathrm{PbO}-\mathrm{V}_{2} \mathrm{O}_{5}$ glass system has been extensively studied and the crystal structure of $\mathrm{PbV}_{2} \mathrm{O}_{6}$ or $\mathrm{PbV}_{2} \mathrm{O}_{7}$ Phases are known [1,2]. This is an ideal system to study the effect of doping or substitution on the physical properties can be explained to the possible changes in the glass structure especially when doping or substitution is carried out at specific composition for which structural information is available. Recently Ramesh and Sastry carried out the work in the field of semi conducting glasses to understand the effect of different oxide groups in the place of $\mathrm{PbO}$ in $\mathrm{PbO}-\mathrm{V}_{2} \mathrm{O}_{5}$ glass system chosen at the eutectic composition i.e. 1:1 molar ratio with the substitution of $\mathrm{ZnO}, \mathrm{CuO}$ and $\mathrm{TiO}_{2}$ in the place of $\mathrm{PbO}$ of $\mathrm{PbO}-\mathrm{V}_{2} \mathrm{O}_{5}$ eutectic glass composition which can be represented as follows, by the general formula XMO:(50-x)PbO: $50 \mathrm{~V}_{2} \mathrm{O}_{5}$, where MO is the substituted metal oxide [3-6]. In all compositions $\mathrm{x}$ varied as $0,5,10$ and 15 mole\%. Recently Bhujanga Rao et al. [7,8] reported the studies to CdO substituted glass systems with similar compositional variations. In the present paper we reported the results of DC Electrical Conductivity, thermoelectric power and ESR studies of CdO substituted lead Vanadate glass systems in the crystalline form compared with glass form in

*Corresponding author. the range of 0 to 15 mole\%.

Glasses of the following compositions xCdO:(50-x) $\mathrm{PbO}: 50 \mathrm{~V}_{2} \mathrm{O}_{5}$ (where $\mathrm{x}=0$, 5, 10 and 15 mole\%) have been prepared using high purity reagent grade materials.

\section{Experimental Work}

The chemicals were weighted in required molar ratio for each batch of composition using an electrical balance (Dhona Model 200D) of 0.0001 gm accuracy. Each batch of composition was taken in agate mortar and was mixed thoroughly for several hours for homogeneity. This mixture whose total weight was around $2 \mathrm{gm}$ for each batch of composition was taken in a glazed silica crucible with lid and was melted in an electrical furnace kept at a temperature of $950^{\circ} \mathrm{C}-1000^{\circ} \mathrm{C}$ range. The completely melted samples were periodically stirred for homogeneity and were kept at that state for nearly 15 minutes before quenching. The melts were quenched on a large stainless steel block maintained at room temperature $\left(30^{\circ} \mathrm{C}\right)$ and constituting of $9 \mathrm{~mm}$ cylindrical cavities to get samples of cylindrical shape of 2 to $3 \mathrm{~mm}$ width. The glass samples were annealed at $150^{\circ} \mathrm{C}$ below the glass transition temperature for nearly 2 hours. The annealed samples were washed with very fine lapping papers. The samples were washed with acetone and dried. 
For the samples used for conductivity measurements a gold coating was deposited on both the polished surfaces using a thin film coating unit. (Model JFC 1000 of JEOL). The $T_{g}$ (Glass transition temperature), $T_{c}$ (Crystalline Temperature) and $T_{m}$ (Melting Point Temperature) for glass systems were studied by thermo gravimetric or differential scanning calorimetric techniques and these values has been reported [7]. The as prepared glass systems were annealed at temperatures corresponding to their of crystalline temperature for four hours for perfect crystalline form by confirming through X-Ray diffraction. Conductivity, Thermo power studies when carried out on such poly-crystalline materials which were obtained in the form of discs of 9 or $10 \mathrm{~mm}$ diameter and 3 or $4 \mathrm{~mm}$ thickness. In the present studies ESR spectra were recorded using JEOL (FE-3X) ESR Spectrometer operated at X-band $(9.3 \mathrm{GHz})$ and employing a $100 \mathrm{KHz}$ field modulation.

\section{Results and Discussion}

The X-ray diffractograms showing perfect amorphous nature of the samples. The X-ray diffractograms for the samples annealed at $623 \mathrm{~K}$ which corresponds to the first crystallization temperature of the samples and evident from X-ray diffractograms which are perfectly crystalline after the heat treatment. X-ray diffractograms were recorded for all the samples are shown in the Figure 1. The DSC pattern for these samples has been reported [7]. It can be seen that there is a gradual increase in the glass transition temperature as $\mathrm{CdO}$ is substituted for $\mathrm{PbO}$. The crystallization peak observed around $324^{\circ} \mathrm{C}$ gradually broadens so does the crystallization peak observed around $435^{\circ} \mathrm{C}$. The crystallization peak observed around $324^{\circ} \mathrm{C}$ moves to higher temperature as the $\mathrm{CdO}$ substitution increases from 5 mole\% to 15 mole\%. There is also a simultaneous decrease in enthalpy (heat released) from 66 $\mathrm{J} / \mathrm{gm}$ to $40 \mathrm{~J} / \mathrm{gm}$ and also the crystallization peak broadens as CdO substitution increases. This is probably an indication of the decrease in the glass forming ability of the compound as $\mathrm{CdO}$ substitution for $\mathrm{PbO}$ is increased from 5 mole\% to 15 mole\%.

In the present investigations on DC Electrical conductivity studies were carried out by using a two probe technique. The resistance of the sample was carried out by using a Keithlay (Model 614) digital electrometer. The conductivity of the sample was calculated from knowledge of the geometry of the sample using the expression

$$
\sigma=\frac{1}{R_{x}}\left(\frac{t}{A}\right)
$$

where $R_{x}$ is the Resistance, $t$ is the thickness and $A$ is the area of the sample.

The logarithmic DC conductivity for amorphous sam- ples as a function of reciprocal of temperature is shown in the Figure 2.

The activation energies obtained by fitting the samples to the Mott's equation are given in the Table 1 [9-13].

$$
\sigma=\sigma_{0} e^{-W / K T}
$$

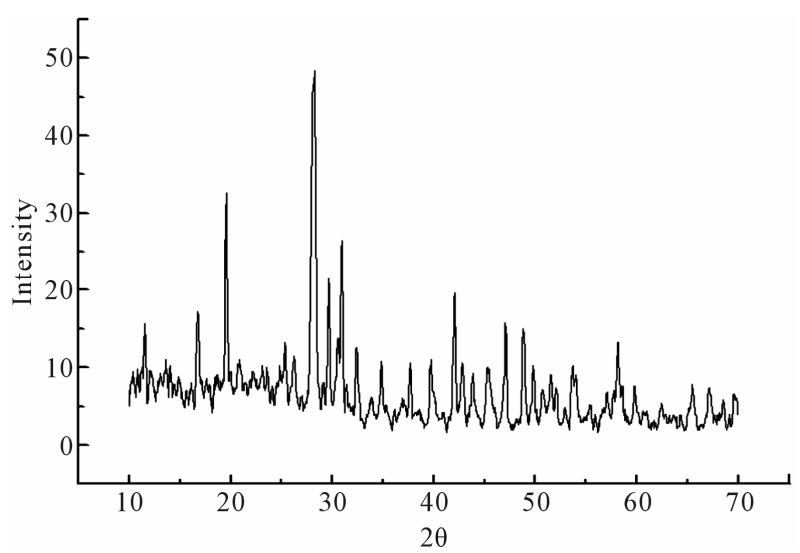

(a)

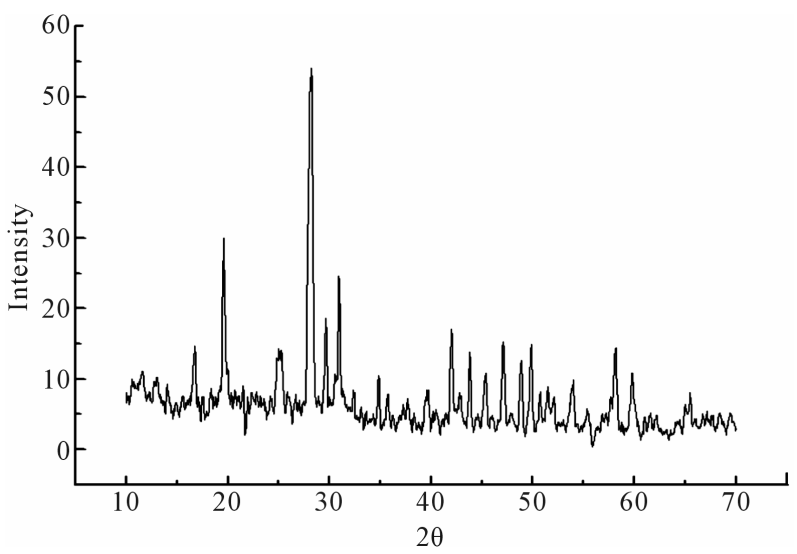

(b)

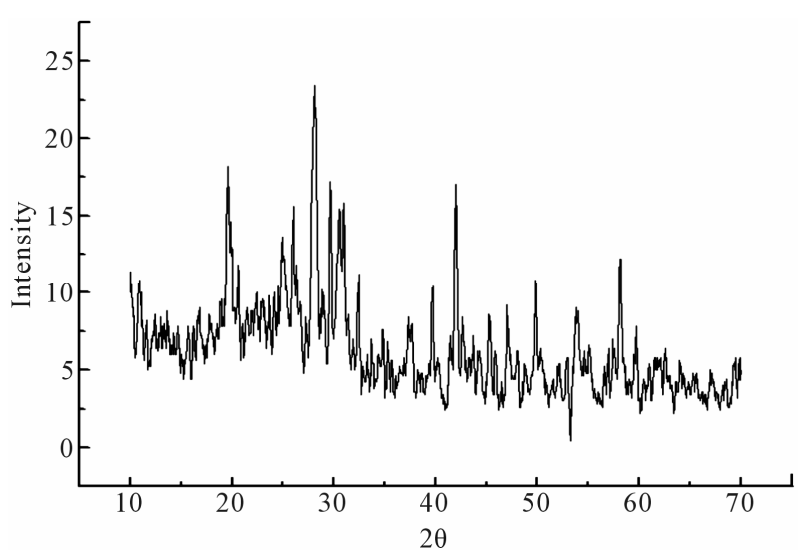

(c)

Figure 1. X-ray diffractograms of $x C d O:(50-x) P b O: 50 V_{2} \mathrm{O}_{5}$ devitrified system. (a) $x=5 \mathrm{~mol} \%$; (b) $x=10 \mathrm{~mol} \%$; (c) $x=$ 15 mol\%. 


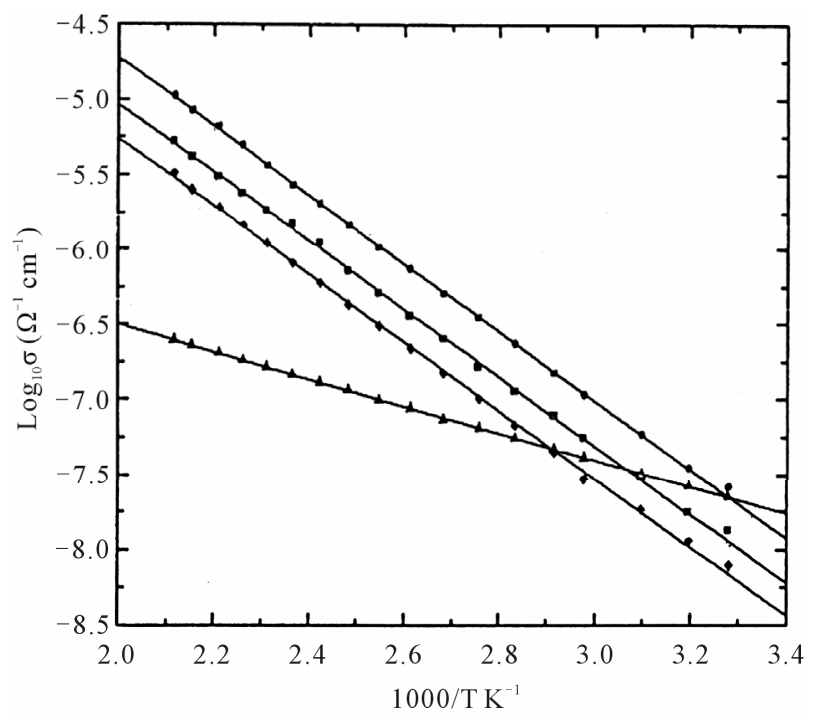

Figure 2. Temperature depends of the logarithmic conductivity of xCdO:(50-x)PbO:50 $\mathrm{V}_{2} \mathrm{O}_{5}$ glass system. $\sim \mathrm{x}=\mathbf{0}$ mol\%; $\forall=5 \mathrm{~mol} \% ; \quad \mathrm{x}=10 \mathrm{~mol} \% ; \bullet x=15 \mathrm{~mol} \%$.

where $\sigma_{0}$ is the pre-experimental factor;

$W$ is the activation energy;

$K$ is the Boltzmann constant;

$T$ is the temperature in Kelvin.

The DC conductivity obtained in the crystalline samples is shown in the Figure $\mathbf{3}$ the activation energies obtained are shown in the Table $\mathbf{1 .}$

The chemical analysis data suggests that is not much change in $\mathrm{V}^{4+}$ concentration in crystalline samples when compared to the amorphous system. At a given temperature the conductivity increases as $\mathrm{CdO}$ substitution increases in these glass systems. At around $303 \mathrm{~K}$, the conductivity of 5 mole\% of CdO substituted sample is less than that of the pure sample. But as temperature increases the conductivity of all the samples increases and is greater than that of the pure sample. As per the reported literature [7] 5 mole\% CdO substituted sample $\mathrm{V}^{4+}$ ion concentration is less than that of the pure sample. But the $\mathrm{V}^{4+}$ ion concentration increases as $\mathrm{CdO}$ concentration increases. The CdO substituted glasses posses higher activation energy for conductivity when compared to $50 \mathrm{PbO}: 50 \mathrm{~V}_{2} \mathrm{O}_{5}$ glass system.

Conductivity data at temperatures higher than room temperature can be fitted to Mott's phonon assisted polaron hopping model. The conduction mechanism in all the glass systems studied can be shown to be due to highly localized small polarons hopping non-adiabaticcally between different sites.

It has been observed in literature that DC conductivity in vanadate glasses with conventional network formers like $\mathrm{GeO}_{2}$ can be explained by adiabatic hopping process and Mott's variable range hopping model can be fitted to conductivity data below $100 \mathrm{~K}$ [14-16]. In sharp contrast,

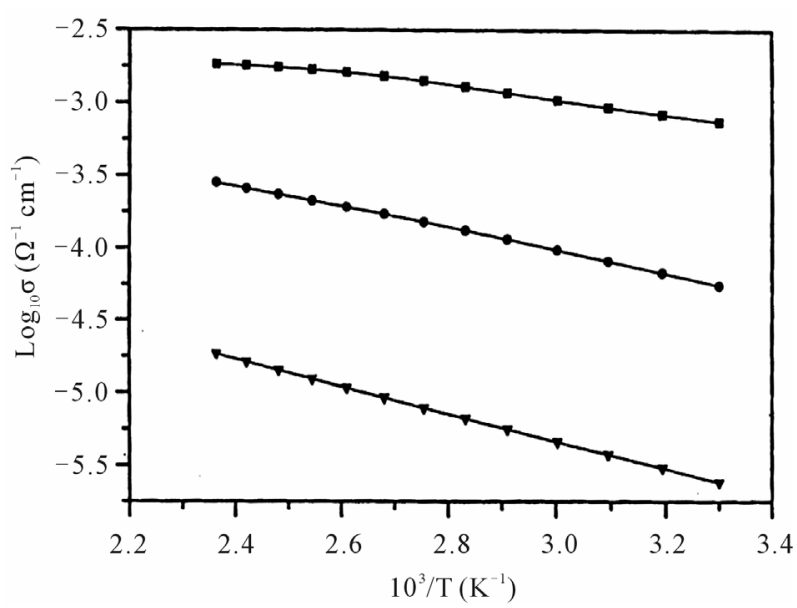

Figure 3. Temperature dependence of the logarithmic conductivity of xCdO:(50-x)PbO:50 $\mathrm{V}_{2} \mathrm{O}_{5}$ crystalline system. $x=5 \% ; \bullet x=10 \% ; \nabla x=15 \%$.

Table 1. Activation energies obtained by fitting the high temperature data to Mott's model for CdO substituted lead vanadate glass and crystalline forms.

\begin{tabular}{ccccc}
\hline \multicolumn{2}{c}{ Glass Composition (Mole\%) } & \multicolumn{2}{c}{$\mathrm{W}(\mathrm{eV})$} \\
\hline $\mathrm{V}_{2} \mathrm{O}_{5}$ & $\mathrm{PbO}$ & $\mathrm{CdO}$ & Glass Form & Crystalline Form \\
\hline 50 & 50 & 0 & 0.175 & 0.175 \\
50 & 45 & 5 & 0.450 & 0.02331 \\
50 & 40 & 10 & 0.452 & 0.03216 \\
50 & 35 & 15 & 0.455 & 0.03549 \\
\hline
\end{tabular}

the conductivity data of lead vanadate glasses [xPbO: (100-x) $\mathrm{V}_{2} \mathrm{O}_{5}$, $\mathrm{x}$ varied between 10 to 50 mole\%] can be explained by non-adiabatic hopping process and variable range hopping model is found to be applicable even at room temperature (about $300 \mathrm{~K}$ ) [17]. The present studies in CdO substituted lead vanadate glasses are consistent with the latter observations.

In $\mathrm{CdO}$ glass systems the conductivity decreases to a value less than that observed in the un-substituted sample at $300 \mathrm{~K}$ for 5 mole\% substitution. However conductivity increases as temperature increases and is characterized by higher activation energy when compared to the pure sample. The decrease in conductivity in these systems is consistent with the decrease in $\mathrm{V}^{4+}$ ion concentration as concentration of substitution is increased. The conductivity mechanism in these systems also can be explained by Mott's phonon assisted non-adiabatic polaron hopping process.

In the reported studies of electrical properties of lead vanadate glasses [17], it has been observed that conductivity decreases (at a given temperature) as $\mathrm{V}_{2} \mathrm{O}_{5}$ concentration is decreased in the glass composition. In the present studies $\mathrm{V}_{2} \mathrm{O}_{5}$ concentration has been kept constant 
(at 50 mole\%) and $\mathrm{PbO}$ concentration has been reduced by equivalent amounts of $\mathrm{CdO}$ substitution. It is interesting that such substitution in the place of network modifier has affected $\mathrm{V}^{4+}$ concentration and hence conductivity. It is also interesting that even though the network former $\left(\mathrm{V}_{2} \mathrm{O}_{5}\right)$ concentration has been kept constant there has been a considerable increase in the activation energy when $\mathrm{PbO}$ has been replaced by $\mathrm{CdO}$. Hence the increased conductivity may be due to increased hopping. The decrease in activation energies in crystalline samples is in accordance with this observation.

In amorphous samples indicates that the Seebeck coefficient is negative for all the samples at room temperature and changes to positive at temperatures higher than the room temperature. This indicates that the conduction is predominantly n-type at low temperature and p-type at higher temperatures.

The temperature depends of the Thermo Electric power above the room temperature for the amorphous samples are shown in the Figure 4. The thermo electric power in the crystalline samples is also measured as a function of temperature and is shown in the Figure 5.

In the crystalline samples the thermo electric power measurements indicate that the seebeck coefficient is positive even at room temperature as well as higher temperature as well as higher temperatures. This indicates that the crystalline materials are predominantly p-type even at room temperature. The thermo electric measurements were fitted to Heike's formula [18] as below:

$$
S=\left(\frac{K_{B}}{e}\right)\left\{\ln \left(\frac{C}{(1-C)}\right)+\alpha^{\prime}\right\}
$$

According to Heike's, $\alpha^{\prime}=\frac{\Delta S^{\prime}}{K_{B}}$

where $\Delta S^{\prime}$ is change in entropy of the system due to the presence of a charge carrier. The $\alpha^{\prime}$ values obtained in the amorphous and crystalline systems at room temperature are given in Table 2.

Austin and Mott [19] suggested that $\alpha^{\prime} \geq 2$ for large polaron and is $\alpha^{\prime}<1$ for small polaron. Hence this observation we can assume that conduction in amorphous samples is predominantly due to formation of large polaron.

The presence of $\mathrm{V}^{4+}$ (electron spin $\mathrm{S}=1 / 2$ and nuclear spin $\mathrm{I}=7 / 2$ and electron ground state $3 \mathrm{~d}^{1}$ ) in vanadate glasses gives rise to good electron spin resonance (ESR) spectra which give information regarding $\mathrm{V}^{4+}$ local symmetry [20]. The $\mathrm{V}^{4+}$ generally exists in six coordinated distorted octahedral symmetry, the degree of distortion being a function of the composition, the nature of phases present and thermal treatment of the glass system [21]. The $\mathrm{V}^{4+}$ ion is assumed to be present in the form of $\mathrm{VO}^{2+}$ molecular ion or radical whose ground state has

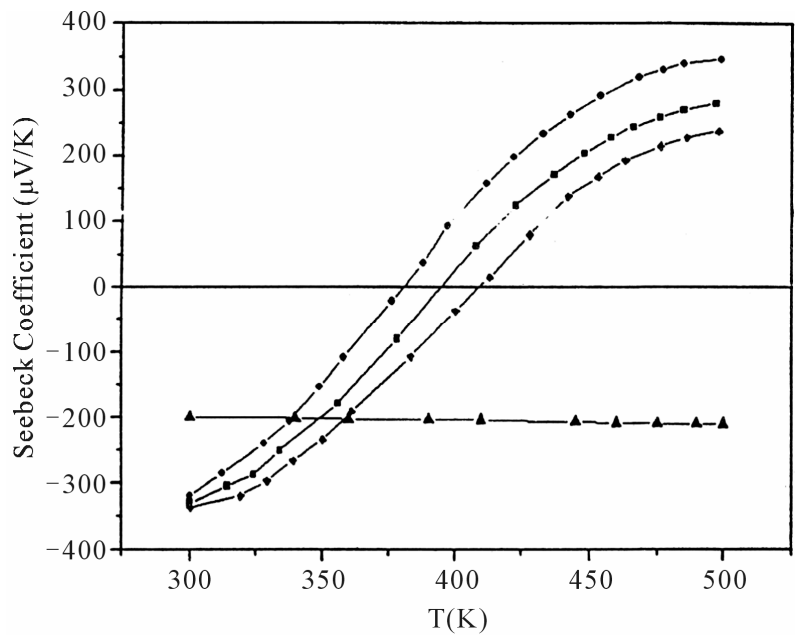

Figure 4. Temperature dependence of the thermoelectric power of xCdO:(50-x)PbO:50 $\mathrm{V}_{2} \mathrm{O}_{5}$ glass system. $\triangle \mathrm{x}=\mathbf{0}$ mol\%; $\checkmark \mathrm{x}=5 \mathrm{~mol} \%$; $\square \mathrm{x}=10 \mathrm{~mol} \%$; $\bullet \mathrm{x}=15 \mathrm{~mol} \%$.

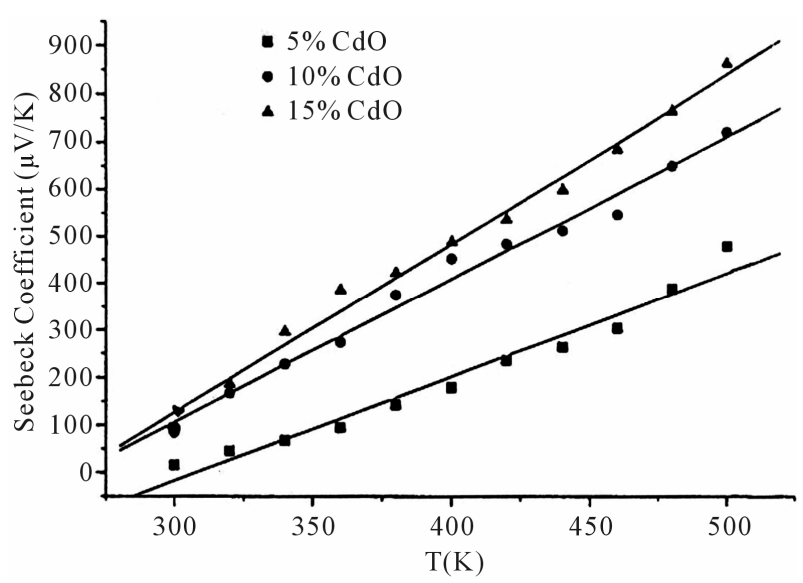

Figure 5. Temperature dependence of the thermoelectric power of xCdO:(50-x)PbO: $50 \mathrm{~V}_{2} \mathrm{O}_{5}$ crystalline form.

Table 2. Parameters obtained from the analysis of Thermo electric power for xCdO:(50-x)PbO: $50 \mathrm{~V}_{2} \mathrm{O}_{5}$ glass and crystalline systems at $300 \mathrm{~K}$.

\begin{tabular}{|c|c|c|c|c|c|}
\hline \multicolumn{3}{|c|}{ Glass Composition (mole\%) } & \multirow{2}{*}{$\begin{array}{c}\text { Seebeck } \\
\text { coefficient } \\
(\mu \mathrm{V} / \mathrm{K})\end{array}$} & \multirow{2}{*}{$\begin{array}{c}\alpha^{\prime} \\
\text { Glass form }\end{array}$} & \multirow{2}{*}{$\begin{array}{c}\alpha^{\prime} \\
\text { Crystalline } \\
\text { form }\end{array}$} \\
\hline $\mathrm{V}_{2} \mathrm{O}_{5}$ & $\mathrm{PbO}$ & $\mathrm{CdO}$ & & & \\
\hline 50 & 45 & 5 & -337 & 0.15 & 4.249 \\
\hline 50 & 40 & 10 & -330 & 0.22 & 5.551 \\
\hline 50 & 35 & 15 & -319 & 0.31 & 5.519 \\
\hline
\end{tabular}

been described by Ballhausen and Gray [22]. The radical $\mathrm{VO}^{2+}$ or $\mathrm{V}^{4+}$ has been studied in several vanadate or $\mathrm{V}_{2} \mathrm{O}_{5}$ containing glass systems [23]. H. El Makami et al. could identify two different $\mathrm{V}^{4+}$ ions in vanadium borate glasses [20]. No such phase separation has been reported in 50PbO: $50 \mathrm{~V}_{2} \mathrm{O}_{5}$ glass system [21]. In the present studies ESR spectra recorded in CdO substituted lead vanadate 
glass system have yielded interesting results which are discussed below. The ESR spectra of CdO substituted lead vanadate glass and crystalline systems are shown in Figures 6 and 7.

The $g$ and $A$ values observed in the present system are very similar to those reported for $\mathrm{V}^{4+}$ ion in vanadate glass systems [21] and given in the Tables $\mathbf{3}$ and $\mathbf{4}$. However a considerable variation is observed in the ESR spectra obtained in the devitrified samples. The CdO substituted lead vanadate sample even after devitrification there seems to be some residual hyperfine structure being present. It has been observed that the ESR spectra of crystalline semi-conducting glasses show a single ESR line due to the collapse of hyperfine interaction which results from increased hopping of electron from $\mathrm{V}^{4+}$ sites to $\mathrm{V}^{5+}$ sites. Thus the increased conductivity of the crystalline samples averages or reduces the hyperfine interaction. It can be seen from the conductivity studies that conductivity is reduced in CdO substituted samples. A similar result was observed by Ramesh in $\mathrm{ZnO}$ substituted lead vanadate glass system and it has been observed that there is some residual hyperfine interaction in $\mathrm{ZnO}$ substituted samples [24]. But in CdO substituted samples, the residual hyperfine interaction is more pronounced.

ESR spectra of CdO sample of 15 mole\% exhibits higher $\mathrm{V}^{4+}$ ion concentration. Hence in line with these observations it can be seen that the ESR spectrum of 15 mole\% of CdO containing sample is relatively less resolved probably due to broadening of the ESR lines due to the presence of higher concentration of $\mathrm{V}^{4+}$ ion.

\section{Conclusions}

It has been observed that there is a rem arkable improvement in the conductivity of the crystalline samples

Table 3. ESR parameters of xCdO (50-x)(PbO):50( $\left(\mathrm{V}_{2} \mathrm{O}_{5}\right)$ glass system at $300 \mathrm{~K}$.

\begin{tabular}{ccccccc}
\hline \multicolumn{3}{c}{ Compositions (mol\%) } & \multicolumn{4}{c}{$300 \mathrm{~K}$} \\
\hline $\mathrm{V}_{2} \mathrm{O}_{5}$ & $\mathrm{PbO}$ & $\mathrm{CdO}$ & $\mathrm{g}_{\|}$ & $\mathrm{g}_{\perp}$ & $\mathrm{A}_{\|}$ & $\mathrm{A}_{\perp}$ \\
\hline 50 & 45 & 5 & 1.924 & 1.962 & 174.1 & 57.5 \\
50 & 40 & 10 & 1.926 & 1.964 & 173.0 & 56.9 \\
50 & 35 & 15 & 1.924 & 1.964 & 172.0 & 52.5 \\
\hline
\end{tabular}

Table 4. ESR parameters of xCdO:(50-x)(PbO):50( $\left(\mathrm{V}_{2} \mathrm{O}_{5}\right)$ devitrified system at $300 \mathrm{~K}$.

\begin{tabular}{cccc}
\hline \multicolumn{3}{c}{ Compositions (mol\%) } & g value \\
\hline $\mathrm{V}_{2} \mathrm{O}_{5}$ & $\mathrm{PbO}$ & $\mathrm{CdO}$ & $300 \mathrm{~K}$ \\
50 & 45 & 5 & 1.939 \\
50 & 40 & 10 & 1.946 \\
50 & 35 & 15 & 1.953 \\
\hline
\end{tabular}

when compared to their amorphous counter parts. There is also remarkable decrease in the activation energy for conduction in the annealed and devitrified samples when compared to amorphous samples. The thermo electric power measurements indicates that the amorphous samples are n-type at room temperature where as the crystalline samples are p-type at room temperature. The thermo electric power measurement also indicates the formation of large polar on in the crystalline samples.

ESR spectra of crystalline system shows a single ESR line due to the collapse of hyperfine interaction which results from increased hopping of electron from $\mathrm{V}^{4+}$ sites to $\mathrm{V}^{5+}$ sites. Thus the increased conductivity of the crystalline samples averages or reduces the hyperfine interaction. The increase in electrical conductivity in the crystalline samples can be attributed to the increased in electron hopping on $\mathrm{V}^{4+}$ or $\mathrm{V}^{5+}$ sites. The increase in the order
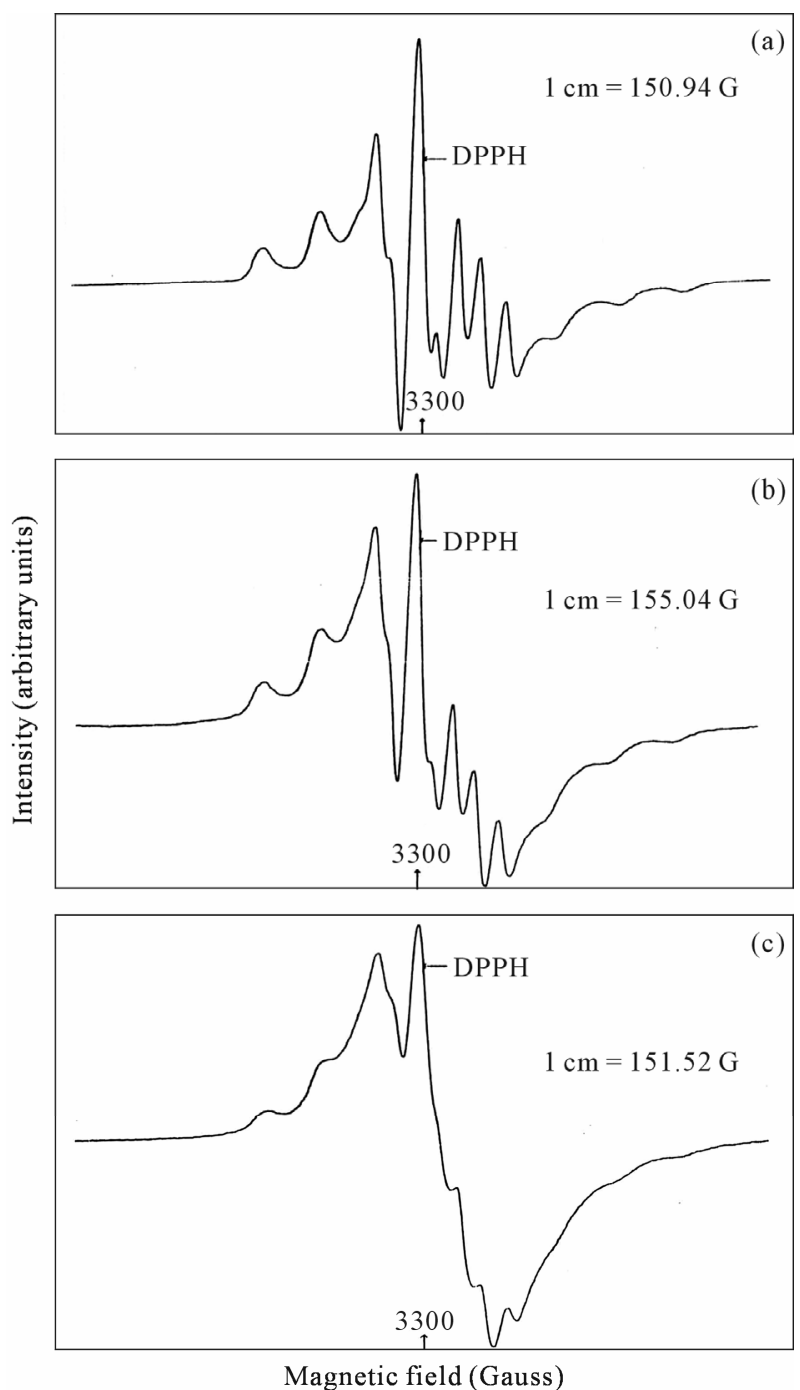

Figure 6. ESR spectra of xCdO:(50-x)PbO:50 $\mathrm{V}_{2} \mathrm{O}_{5}$ glass system at $300 \mathrm{~K}$. (a) $x=5 \mathrm{~mol} \%$; (b) $x=10 \mathrm{~mol} \%$; (c) $x=15$ mol\%. 

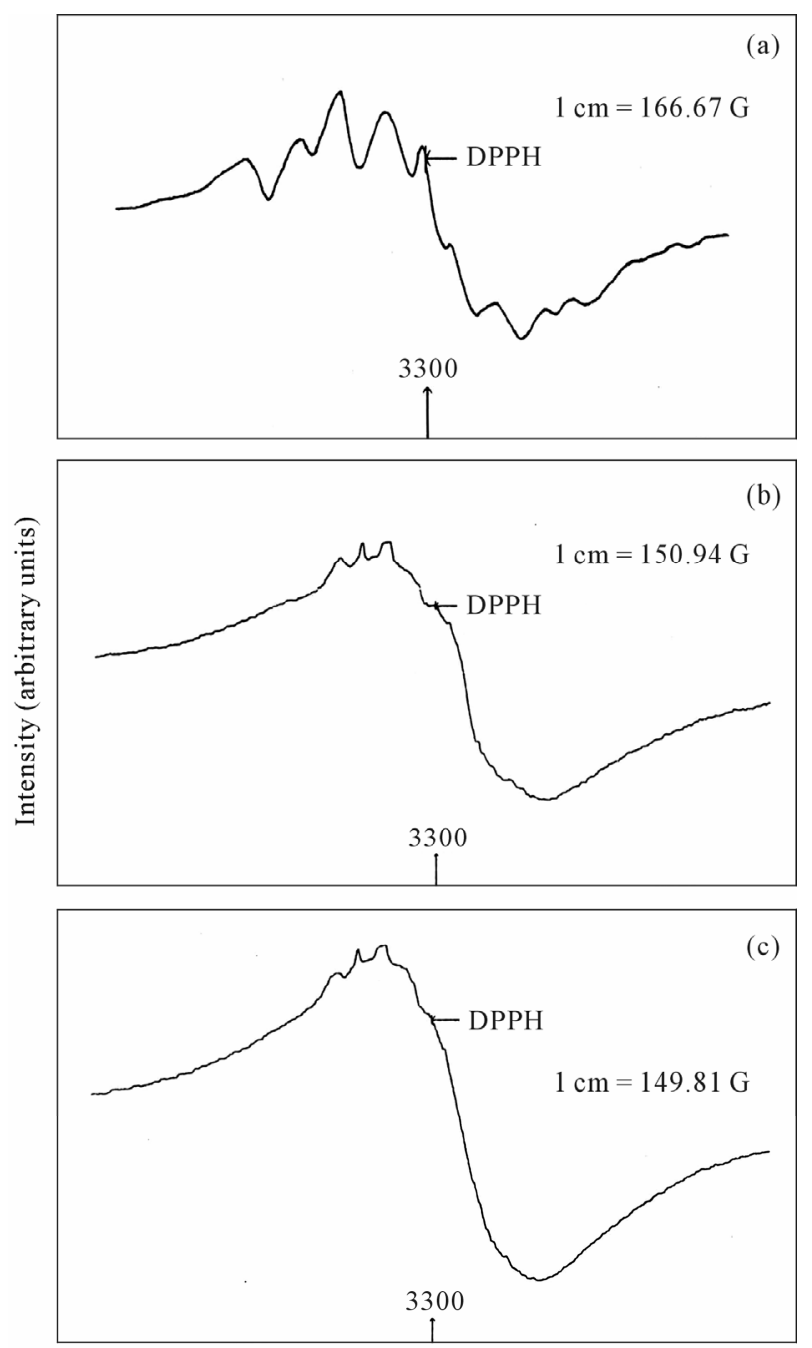

Magnetic field (Gauss)

Figure 7. ESR spectra of $x C d O:(50-x) P b O: 50 \mathrm{~V}_{2} \mathrm{O}_{5}$ crystal system at 300 K. (a) $x=5 \mathrm{~mol} \%$; (b) $x=10 \mathrm{~mol} \%$; (c) $x=15$ mol\%.

of the structural units in the crystalline samples causes the increased probability of electron hopping from $\mathrm{V}^{4+}$ to $\mathrm{V}^{5+}$ sites, and hence to increase in conductivity and decreased activation energies.

\section{REFERENCES}

[1] A. E. Owen, "Electronic and Structural Properties of Amorphous Semi Conductors,” Academic Press, London, 1973.

[2] P. W. Anderson, "Absence of Diffusion in Certain Random Lattices,” Physical Review, Vol. 109, No. 5, 1958, pp. 1492-1505. doi:10.1103/PhysRev.109.1492

[3] K. V. Ramesh and D. L. Sastry, “Transport Properties of ZnO Substituted Lead Vanadate Glass System at Eutectic Composition,” Materials Science and Engineering: B, Vol. 126, 2006, pp. 66-73. doi:10.1016/j.mseb.2005.08.111

[4] K. V. Ramesh and D. L. Sastry, “Temperature-Dependent
Thermoelectric Power of $\mathrm{CuO}, \mathrm{ZnO}$ and $\mathrm{TiO}_{2}$ Substitute for PbO in Eutectic Lead Vanadate Glass System,” International Journal of Modern Physics B, Vol. 18, No. 25, 2004, pp. 3327-3341. doi:10.1142/S0217979204026482

[5] K. V. Ramesh and D. L. Sastry, "DC Electrical Conductivity, Thermoelectric Power Measurements of $\mathrm{TiO}_{2}$-Substituted Lead Vanadate Glasses,” Physica B: Condensed Matter, Vol. 387, No. 1-2, 2007, pp. 45-51. doi:10.1016/j.physb.2006.03.026

[6] K. V. Ramesh and D. L. Sastry, "IR and ESR Studies of $\mathrm{CuO}$ Substituted for $\mathrm{PbO}$ in Eutectic Lead Vanadate Glass System,” Journal of Non-Crystalline Solids, Vol. 352, No. 50-51, 2006, pp. 5421-5428. doi:10.1016/j.jnoncrysol.2006.08.017

[7] C. B. Rao, K. V. Ramesh and D. L. Sastry, "Anomalous Temperature Variation of Thermoelectric Power in CdO and $\mathrm{Ag}_{2} \mathrm{O}$ Substituted Lead Vanadate Glass System," Physica B: Condensed Matter, Vol. 382, No. 1-2, 2006, pp. 81-85. doi:10.1016/j.physb.2006.02.002

[8] C. B. Rao, "Physical Properties of CdO, $\mathrm{Ag}_{2} \mathrm{O}$ and $\mathrm{TeO}_{2}$ Substituted for $\mathrm{PbO}$ in Eutectic Lead Vanadate Glass System,” Ph.D. Thesis, Andhra University, Visakhapatnam, India, 2005.

[9] A. Ghosh and B. K. Chaudari, "DC Conductivity of $\mathrm{V}_{2} \mathrm{O}_{5}-\mathrm{Bi}_{2} \mathrm{O}_{3}$ Glasses,” Journal of Non-Crystalline Solids, Vol. 83, No. 1-2, 1986, pp. 151-161. doi:10.1016/0022-3093(86)90065-7

[10] S. Mandal and A. Ghosh, "Electrical Properties of Lead Vanadate Glasses,” Physical Review B, Vol. 49, No. 5, 1994, pp. 3131-3135. doi:10.1103/PhysRevB.49.3131

[11] N. F. Mott and E. A. Davis, "Electronic Processes in Non -Crystalline Materials,” 2nd Edtion, Clarendon Press, Oxford, 1979.

[12] E. A. Davis and N. F. Mott, "Conduction in Non-Crystalline Systems V. Conductivity, Optical Absorption and Photoconductivity in Amorphous Semiconductors," Philosophical Magazine, Vol. 22, No. 179, 1970, pp. 903922. doi:10.1080/14786437008221061

[13] N. F. Mott, "Conduction Glasses Containing Transition Metal Ions," Journal of Non-Crystalline Solids, Vol. 1, No. 1, 1968, pp. 1-28. doi:10.1016/0022-3093(68)90002-1

[14] M. Sayer and A. Mansingh, “Transport Properties of Semiconducting Phosphate Glasses,” Physical Review B, Vol. 6, No. 12, 1972, pp. 4629-4643.

[15] A. Ghosh, "Transport Properties of Vanadium Germanate Glassy Semiconductors,” Physical Review B, Vol. 42, No. 9, 1990, pp. 5665-5676. doi:10.1103/PhysRevB.42.5665

[16] C. H. Chung, J. D. Mackenzie and L. Murawski, "Electrical Properties of Semiconducting Oxide Glasses," Revue de Chimie Minerale, Vol. 16, 1979, pp. 308-327.

[17] S. Mandal and A. Ghosh, "Electrical Properties of Lead Vanadate Glasses,” Physical Review B, Vol. 49, No. 5, 1994, pp. 3131-3135. doi:10.1103/PhysRevB.49.3131

[18] R. R. Heikes, “Thermoelectricity,” Interscience, New York, 1961.

[19] I. G. Austin and N. F. Mott, "Polarons in Crystalline and Non-Crystalline Materials,” Advances in Physics, Vol. 18, 
No. 17,1969 , pp. 41-53.

doi:10.1080/00018736900101267

[20] H. El Mkami, B. Deroide, N. Abidi, P. Rumori and J. V. Zanchetta, "ESR Study and DC Conductivity of Binary Glasses of the System $\left(\mathrm{V}_{2} \mathrm{O}_{5}\right)_{\mathrm{x}}\left(\mathrm{B}_{2} \mathrm{O}_{3}\right)_{1-\mathrm{x}}$," Physics and Chemistry of Glasses, Vol. 38, 1997, pp. 137-145.

[21] F. Momo, A. Sotgin, E. Baicchi, M. Bettinelli and A. Montenero, "ESR Study of the Equimolar $\mathrm{PbO}-\mathrm{V}_{2} \mathrm{O}_{5}$ System," Journal of Materials Science, Vol. 17, No. 11, 1982, pp. 3221-3226. doi:10.1007/BF01203486
[22] C. J. Ballhausen and H. B. Grey, "The Electronic Structure of the Vanadyl Ion,” Inorganic Chemistry, Vol. 1, No. 1, 1962, pp. 111-132. doi:10.1021/ic50001a022

[23] L. D. Bogomolov, "The Effects of Copper Impurity in Vanadate and Tungsten-Phosphate Glasses,” Journal of NonCrystalline Solids, Vol. 30, 1979, pp. 378-383.

[24] K. V. Ramesh, "Thermal, Electrical and Spectroscopic Studies of $\mathrm{CuO}, \mathrm{ZnO}$ and $\mathrm{TiO}_{2}$ Substituted for $\mathrm{PbO}$ in Eutectic Lead Vanadate Glass System,” Ph.D. Thesis, Andhra University, Visakhapatnam, India, 2000. 